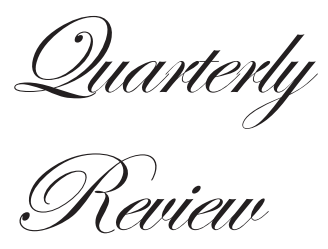

\title{
Difficult to treat spontaneous bacterial peritonitis
}

\author{
Shalimar, Subrat Kumar Acharya
}

\section{ABSTRACT}

Department of Gastroenterology and Human Nutrition Unit, All India Institute of Medical

Science, New Delhi 110029, India

\section{Correspondence:}

Dr. Subrat Kumar Acharya

Email:subratacharya2004@yahoo.com
Spontaneous bacterial peritonitis (SBP) is the most frequent infection in patients with cirrhosis. It is associated with high mortality at admission and its occurrence alters the natural course with a high 1 year mortality. Presence of $>250$ polymorphonuclear cell $(\mathrm{PMN}) / \mathrm{mm}^{3}$ in the ascitic fluid is diagnostic of SBP. SBP is usually treated with IV antibiotics using third generation cephalosporins and fluoroquinolones. However, despite effective initial treatment subsequent recurrence of SBP with its accompanying mortality has resulted in use of long term antibiotic prophylaxis and such patients are recommended for liver transplant. An increased frequency of multidrug resistant bacterial SBP has recently been recognised with use of prophylaxis and is associated with enhanced mortality. Further, cirrhotics get repeated hospitalisation and ICU care leading to nosocomial infection causing SBP. Therefore, frequency of multidrug resistant bacteria induced SBP among the above settings has increased and the relative risk (RR) of mortality with bacterial resistance has been estimated to be 4 times higher than in patients with SBP without bacterial resistance. Therapeutic approach in such patients at present is a clinical challenge and they are difficult to treat patients. Therefore, SBP can be categorized into community acquired and nosocomial/ multidrug resistant SBP.

KEYWORDS: SBP, difficult to treat, nosocomial SBP

\section{Introduction}

Spontaneous bacterial peritonitis (SBP) is defined as 'bacterial infection of ascitic fluid and peritoneum in patients with ascites in absence of any intra abdominal source of infection or malignancy' ${ }^{1-4}$ It is a frequent complication of decompensated cirrhosis with a prevalence of $10-30 \%^{1}$ in hospitalized patients and in about $3.5 \%^{2}$ of out-patients who are usually asymptomatic. Once SBP develops, the prognosis of cirrhosis worsens. The median mortality during first episode of SBP has been reported to be around $30 \%{ }^{3}$ (range 10-50\%) and in such patients the median mortality at 1 year is reported to be about
$66 \%$ (range 30-90\%). Therefore, once a patient recovers from the $1^{\text {st }}$ episode of SBP, he is advised to undergo liver transplant. However, the mortality associated with $1^{\text {st }}$ episode of SBP, has reduced considerably during the last decade due to the awareness and identification of high risk cirrhotics likely to develop SBP, its early diagnosis and effective antibiotic strategy. Recurrence of SBP is high. Therefore primary and secondary prophylaxis using appropriate antibiotics is recommended. However, in such patients, recent recognition of SBP with multidrug resistant bacteria has been associated 
with very high mortality and they are difficult to treat due to the presence of bacteria nonresponsive to community used antibiotics (Clinical course of multidrug resistant SBP has been depicted in Box 1).

Box 1 : Clinical Scenario of multidrug resistant SBPA 45 years old male, decompensated HBV cirrhosis (HBsAg+, $\mathrm{HBeAg}-$, Anti $\mathrm{HBeAb}+$, HBV DNA $>10^{5} \mathrm{IU} / \mathrm{ml}$ ), Child score 9 on treatment with tenofovir, was admitted with upper GI bleed and hemodynamic instability. Post endotherapy, on same day he developed fever and pain abdomen. Investigations revealed haemoglobin $7 \mathrm{gm} / \mathrm{dl}$, TLC $15,000 / \mathrm{mm}^{3}$ (N78L20), Ascitic fluid evaluation: PMN 560/ $\mathrm{mm}^{3}$, SAAG: $1.9 \mathrm{~g} / \mathrm{dl}$ and culture sent. Blood culture was also sent. He was admitted to ICU and started on IV cefotaxime (empirical) and albumin (adjuvant). He improved symptomatically in 2 days, repeat ascitic fluid (48 hours): PMN: $80 / \mathrm{mm}^{3}$, culture was sterile (response). He was discharged after 5 days of IV antibiotics. Norfloxacin was prescribed for prophylaxis, tenofovir continued and he was listed for liver transplant. He was readmitted 6 months later with pain abdomen and Grade I Hepatic Encephalopathy (HE), Child Score 10, serum creatinine $1 \mathrm{mg} / \mathrm{dl}$, MELD 16. He received cefotaxime prior to coming to the hospital. Ascitic fluid evaluation: $400 \mathrm{PMN} / \mathrm{mm}^{3}$, ascitic fluid and blood culture sent on day of admission. He was continued on cefotaxime, he developed fever on day 2 , repeat ascitic fluid cell count (48 hours): $600 \mathrm{PMN} / \mathrm{mm}^{3}$, culture: ESBL +ve E. coli (sensitive to imipenem and meropenem, resistant to cefotaxime, ciprofloxacin and ceftazidime). The antibiotics were changed as per the culture reports and he was started on imipenem. His clinical condition worsened, developed severe sepsis with worsening renal function (creatinine $2.5 \mathrm{mg} / \mathrm{dl}$ ). He developed septic shock and succumbed on day 6 .

The important issues in SBP as a clinical entity include the clinical presentation, diagnosis, risk factors, and management (empirical/specific antibiotic treatment, response evaluation, adjuvant albumin treatment and prophylaxis). The recent documentation of multidrug resistant bacteria causing SBP nonresponsive to recommended empirical antibiotics, and associated high mortality, is the reason why we have used the term 'Difficult to treat SBP' for such cases about which most guidelines are silent. ${ }^{4}$ In this review we will be highlighting the above mentioned issues.

\section{Clinical presentation}

The clinical presentation is variable, is inadequate for diagnosis of SBP and can be categorized into 3 groups: 1) clinical features of peritonitis (e.g. pain and tenderness in the abdomen, fever and increase in gastrointestinal motility) 2) clinical and biochemical deterioration of hepatic and renal function 3) asymptomatic/ aggravation of constitutional symptoms (detected in out-patients in 3-4\% of cirrhotic ascites). Therefore all recommendations (International Ascitic $\mathrm{club}^{5}, \mathrm{AASLD}^{6}$ and $\mathrm{EASL}^{7}$ ) recommend that all cirrhotic patients with ascites should have routine diagnostic paracentesis irrespective of clinical features and suspicion of SBP.

\section{Pathogenesis}

The gut flora and bacterial translocation (BT) play an important role in the pathogenesis of SBP. The translocation of bacteria from the intestine to mesenteric lymph nodes occurs normally. Whenever this physiological phenomenon of BT increases in rate or severity it leads on to bacteremia and subsequent colonization of ascitic fluid. In addition, invasive procedures can lead to nosocomial SBP. E. coli, Klebsiella pneumonia and streptococci are the commonest organisms responsible for SBP. The factors leading to increased $\mathrm{BT}^{8}$ in cirrhosis include: (i) alterations in gut microflora (ii) increased intestinal permeability and (iii) deficiency of local immune response.

\section{Diagnosis}

There is uniform agreement amongst clinicians all over the world that ascitic fluid polymorphonuclear count $(\mathrm{PMN})>250$ / $\mathrm{mm}^{3}$ is adequate to diagnose SBP. ${ }^{6}$ In the event of a traumatic paracentesis (hemorrhagic ascitic fluid) it has been arbitrarily suggested that for each $250 \mathrm{RBC}, 1 \mathrm{PMN}$ needs to be subtracted to assess the accurate number of PMN present in the ascitic fluid. Recently automated counters have been shown to provide results within minutes at low cost.

Leukocyte esterase strips which detect leukocyte esterase in activated PMNs have been tried to provide immediate diagnosis of SBP, but have unacceptable false negative rates ${ }^{9}$. Detection of bacterial DNA in the ascitic fluid using various techniques has been proposed with an aim to identify type of bacteria and therefore possible use of more appropriate antibiotics ${ }^{10}$. However, presence of bacteria in ascitic fluid and 
serum does not necessarily predict the development of SBP and bacterial infection. Further, most studies in SBP have documented gram negative bacteria (predominantly due to $\mathrm{E}$. coli and Klebsiella sp) as the causative agent subsequent to small intestinal bacterial transmigration through mesenteric lymph nodes ${ }^{8}$.

Many other markers have also been described to diagnose SBP which include ascitic fluid $\mathrm{pH}$, lactate, arterial-ascitic fluid lactate gradient, ascitic fluid LDH. However, none of these markers are superior to ascitic fluid PMN count $>250 / \mathrm{mm}^{3}$ in diagnosing SBP. Lactoferrin is released by PMNs in the ascitic fluid. A cut off level of $242 \mathrm{ng} / \mathrm{ml}$, predicts the diagnosis of SBP with $95 \%$ sensitivity and $97 \%$ specificity. ${ }^{11}$ These studies involved a small number of patients; more data is required before incorporating these tests into routine clinical practice.

Ascitic fluid and simultaneous blood culture have been recommended by all recommendations despite the fact that conventional culture may not detect bacteria in about $65 \%$ of patients (culture negative neutrocytic ascites - CNNA). Recently, non radiometric (colorimetric-Bac-T/ALERT) systems have been used to detect bacterial growth in a shorter time ${ }^{12}$. Bed side inoculation of ascitic fluid into blood culture bottles has been documented to improve culture yield in up to $80 \%$ of such patients ${ }^{13}$. Ascitic fluid culture provides a guide to specific treatment. Collation of type of organism and their sensitivity pattern from such patients in a particular set up provides opportunity for selecting appropriate antibiotics. Such information in an individual centre or region is important because, empirical antibiotic therapy is usually recommended once SBP is diagnosed by documenting ascitic fluid PMN > $250 / \mathrm{mm}^{3}$ and culture reports of ascites and blood are usually not available at this time. However a positive blood culture with ascitic fluid culture in patients with SBP would indicate increased bacterial load and therefore may indicate worse prognosis in such patients.

\section{Risk factors}

All cirrhosis patients with ascites do not develop SBP. The development of SBP is associated with poor outcome. Therefore identification of patients at high risk for developing SBP may assist clinicians in monitoring these patients more aggressively. Antibiotics for primary prophylaxis have been found to be beneficial in these high risk patients even though their role remains controversial. These risk factors can be categorized into (i) clinical and biochemical parameters (ii) genotypic factors (iii) iatrogenic factors.

\section{(i) Clinical and biochemical risk factors}

Presence of ascites with ascitic fluid protein of $<1.5 \mathrm{gm} / \mathrm{dl}^{14}$, low ascitic fluid $\mathrm{C} 3{ }^{15}$, poor liver function (each point increase in the MELD score increases the risk of developing SBP by $11 \%)^{16}$, hyperbilirubinemia $(>3.2 \mathrm{mg} / \mathrm{dl})$, low platelets $(<98,000 /$ $\mathrm{mm} 3)^{17}$ and occurrence of variceal bleeding have been identified as risk factors for development of SBP. The cumulative probability of infection during a single hospitalization for bleeding is approximately $36 \% .{ }^{18}$

\section{(ii) Genotypic risk factors}

MCP-1 (Monocyte chemotactic protein-1) ${ }^{19}$, NOD2 ${ }^{20}$ and TLR2 polymorphisms ${ }^{21}$ have been recently described to be more frequently associated with occurrence of SBP. These are well known components of innate immune system in the peritoneal cavity and their suboptimal response to clear infection from the peritoneal cavity subsequent to transmigration of bacteria from small intestine through the mesenteric lymph nodes has been described to be a definitive risk factor for development of SBP.

\section{(iii) Iatrogenic risk factors}

Use of proton pump inhibitors is known to cause small intestinal bacterial overgrowth (SIBO) ${ }^{22}$ which has been identified to be associated with increased risk for developing SBP. Any invasive procedure in patients with cirrhotic ascites may also predispose them for development of SBP.

In contrast, beta-blockers may protect against SBP. A recent meta-analysis included 3 each of RCTs and retrospective studies. There was a statistically significant difference of $12.1 \%$ $(\mathrm{P}<0.001)$ in favour of propranolol in preventing $\mathrm{SBP}^{23}$.

\section{Management}

In the earlier section, early diagnosis of SBP with an aim to institute specific treatment at the earliest has been emphasized. With the establishment of diagnosis of SBP by documenting ascitic fluid PMN $>250 / \mathrm{mm}^{3}$, empirical antibiotic treatment is uniformly recommended as culture results are not available at this time. Recently it has been documented that site of acquisition of the bacteria causing SBP may influence response to antibiotic treatment. Nosocomially acquired SBP (indicated by previous ICU stay, previous antibiotic use, infection acquired by interventions in hospital) in contrast to community 
acquired SBP is more frequently associated with ESBL +ve enterobacteriacae and therefore has been reported to be associated with poor response to empirically recommended antibiotics with subsequent high mortality. Unfortunately, none of the guidelines distinguish the nosocomial and community acquired SBP for the empirical antibiotic approach after the diagnosis.

Nosocomial acquired SBP which is resistant to presently recommended empirical treatment and all patients with SBP having high risk factors for higher mortality (severe liver dysfunction and renal dysfunction at diagnosis of SBP) should be termed as 'Difficult to treat SBP'. This terminology is justified because these subgroups of patients with SBP have been documented to constitute the majority of the patients with treatment failure and consequent increased mortality. Management of both community acquired as well as nosocomial SBP is discussed below.

\section{1) Treatment in community acquired SBP}

In patients with SBP without history of previous hospitalization, interventions or prior antibiotic treatment; Gram -ve bacteria, almost exclusively Enterobacteriacae have been isolated. Even though almost 70 various isolates have been identified in patients with SBP. E. coli and Klebsiella sp constitute most of the isolated bacteria in such patients. Several antibiotics have been used in these patients with similar efficacy, which includes cefotaxime, other $3^{\text {rd }}$ generation cephalosporins, quinolones and amoxycyllin-clavulanate. In patients with GI bleed (an important risk factor for SBP) and in those without associated complications (e.g. encephalopathy, renal dysfunction, shock, ileus etc) oral fluoroquinolones have been shown to provide adequate therapeutic response.

\section{2) Treatment of nosocomial SBP (difficult to treat)}

Even though consensus definition of nosocomial SBP is lacking, most clinicians suggest that nosocomial SBP should be suspected when a patient who is diagnosed to have SBP has a history of ICU stay during the previous 3 months, or was on prophylactic antibiotics for infection or had antibiotic treatment during previous 3 months or had a recent intervention in the hospital setting. Recent reports on SBP (published from 2009$2012)^{24,25}$ indicate that a quarter of organisms isolated from the nosocomial SBP patients are resistant to multiple commonly used antibiotics. Resistance to $3^{\text {rd }}$ generation cephalosporins and quinolones has been documented in $40-50 \%$ of such cases. In some series MRSA has been isolated in about $27 \%$ of the patients $^{26}$. Up to $30 \%$ of the Enterobacteriacae isolated from such patients have been shown to be ESBL positive. These bacteria are resistant to recommended empirical treatment using $3^{\text {rd }}$ generation cephalosporins, fluoroquinolones and amoxicillin-clavulanate and are associated with treatment failure accompanied with high mortality.

A recent metaanalysis indicates that the relative risk (RR) of mortality in such patients with resistant bacteria is 4 times over those without such resistant bacteria ${ }^{27}$. Despite the fact that most guidelines are silent on the choice of empirical antibiotic in such nosocomial SBP patients, local argument based on the available information would suggest use of antibiotics whose sensitivity is preserved in that particular region or centre (this information is usually based on the antibiotic sensitivity pattern available in the particular setup or region). In Europe, the sensitivity of carbapenems and glycopeptides is preserved amongst most Gram -ve bacteria and Gram +ve bacteria respectively. Therefore, certain experts from this region recently have suggested that in nosocomial SBP the $1^{\text {st }}$ line empirical antibiotic of choice should be carbapenem, and should be replaced if necessary with the availability of culture and sensitivity of the ascitic fluid. Figure 1 highlights a simplified approach to management of SBP.

\section{3) Response evaluation}

At the end of 48 hours of antibiotic treatment if ascitic fluid PMN (A-PMN) has not decreased by at least $25 \%$ from the pre-treatment A-PMN value, it is better to change antibiotics based on the culture and sensitivity report of the ascitic fluid which is usually available by this time. However, this recommendation by the International Ascites Club ${ }^{5}$ is arbitrary and is based on the documentation of reduction of A-PMN by $25 \%$ in $>90 \%$ of survivors versus $<70 \%$ in non-survivors with SBP. Further, PMN half life is about 24 hours and therefore lack of adequate reduction in A-PMN at the end of 48 hours of therapy may suggest continuous influx of PMN to the peritoneal cavity due to persistence of infection.

\section{4) Duration of antibiotic therapy}

Antibiotic therapy can be discontinued after A-PMN is $<250 /$ $\mathrm{mm}^{3}$. Comparative studies have reported that 5 days' and 10 days' antibiotic treatment have similar efficacy ${ }^{28}$. Rather, 


\section{Cirrhotic Ascites}

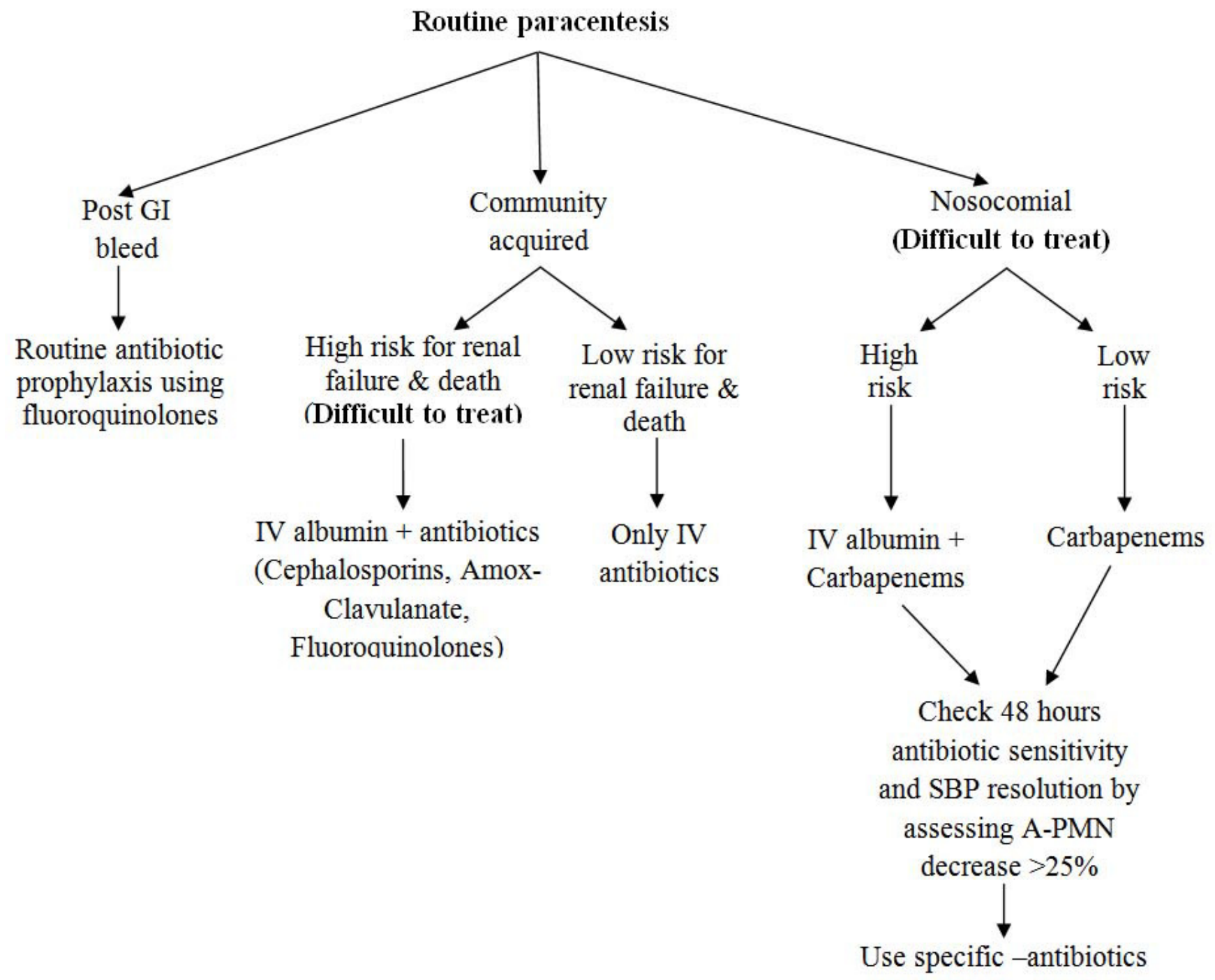

Figure 1: Approach to management of SBP

antibiotic therapy should be guided by A-PMN monitoring after 48 hours of initial institution of antibiotic treatment.

\section{5) Adjuvant use of intravenous albumin infusion (Difficult to treat)}

Multivariate analysis in various studies on SBP have clearly documented independent predictors of mortality which include (i) severity of liver dysfunction indicated by serum bilirubin > $4 \mathrm{mg} / \mathrm{dl}$, (ii) renal dysfunction indicated by blood urea $>30 \mathrm{mg} /$ $\mathrm{dl}$ and serum creatinine $>1.2 \mathrm{mg} / \mathrm{dl}$, (iii) nosocomial source of SBP, (iv) severe sepsis as indicated by positivity of both blood as well as ascitic fluid (suggests increased bacterial load) and (v) hemodynamic instability.
Among all these parameters, severity of liver dysfunction (bilirubin $>4 \mathrm{mg} / \mathrm{dl}$ ) and renal dysfunction (blood urea e" 30 $\mathrm{mg} / \mathrm{dl}$ ) have been identified as predictors of death despite antibiotic treatment in most studies. In such patients intravenous albumin $(1.5 \mathrm{mg} / \mathrm{kg}$ on day $1 \mathrm{and} 1 \mathrm{mg} / \mathrm{kg}$ on day 3$)$ has been found to be of benefit. With use of IV albumin as adjuvant, in these patients the frequency of renal failure has been documented to decrease from $33 \%$ to $10 \%$ and 3 month mortality decreases from $29 \%$ to $10 \%{ }^{29}$. In contrast, the frequency of renal failure and 3 months mortality in those without the high risk parameters for mortality has been reported to be $0-4.7 \%$ and $0-6.5 \%$ respectively and IV albumin as adjuvant to antibiotics did not influence the outcome. However, in unselected patients with SBP, $10 \mathrm{gm}$ of IV albumin/day for 3 
days has been reported to reduce the pro-inflammatory cytokines like IL-6 and TNF-á in blood and ascitic fluid as well as NO in the blood ${ }^{30}$ (which decrease the systemic vascular resistance with consequent up-regulation of plasma renninangiotensin pathway which are believed to cause efferent renal artery vasoconstriction with decrease in GFR). Further studies are therefore needed to evaluate the role of low dose IV albumin in difficult to treat SBP patients.

\section{6) Role of primary and secondary prophylaxis in SBP in view of recently described nosocomial SBP}

Most guidelines and experts suggest use of prophylactic antibiotics in patients of cirrhosis with gastrointestinal bleed and in patients who have recovered from $1^{\text {st }}$ episode of $\mathrm{SBP}^{4}$. For such secondary prophylaxis, strongest evidence exists for norfloxacin $^{31}$. However duration for such prophylaxis is unclear, because most studies provide the duration and follow up of one year. Because liver transplant is recommended in such patients, the relevance of prolonged secondary prophylaxis beyond one year may not be relevant in the western developed country due to better and coordinated access to liver transplantation. The access to liver transplantation in South Asia and in other developing countries is yet limited and therefore, alternative treatment strategies are needed to prevent SBP in the above patients in the long term. Use of rifaximin (a non absorbable antibiotic with efficacy against gram negative enterobacteriacae and lower frequency of resistance), probiotics and antibiotic recycling needs evaluation in these regional areas to evaluate their efficacy in prevention of second episode of SBP.

In contrast to secondary prophylaxis, primary prophylaxis using antibiotics in those patients with high risk for development of SBP (low protein ascites $<1.5 \mathrm{gm} / \mathrm{dl}$ ) remains controversial. Eight randomized control trials (RCTs) have been conducted to evaluate the role of primary prophylaxis in SBP. Two meta-analysis also have been published on this issue with conflicting conclusions. However, a recent meta-analysis which included only three high quality RCTs supports use of quinolones in preventing the first episode of $\mathrm{SBP}^{4}$. Despite these evidences, most experts do not recommend routine use of primary prophylaxis in cirrhosis with low protein ascites, unless an additional risk factor for development of SBP exists (e.g. CTP score $>9$, bilirubin $>3 \mathrm{mg} / \mathrm{dl}$, serum creatinine $>1.2$ $\mathrm{mg} / \mathrm{dl}$, serum sodium $<130 \mathrm{meq} / \mathrm{L}$, blood urea $>25 \mathrm{mg} / \mathrm{dl})^{32}$. The apprehension for development of multi-resistant bacteria has resulted in such a cautious approach.

\section{Conclusions}

- $\quad \mathrm{SBP}$ is a frequent infection in cirrhosis, responsible for 10$30 \%$ of all bacterial infections

- Associated with a high in-hospital mortality (30\%), as well as 1 year mortality of $50-60 \%$

- Clinical judgement does not rule out SBP. All cirrhotic ascites should have diagnostic paracentesis

- High risk patients with SBP can be identified for primary prophylaxis using fluoroquinolones (GI bleed/ low protein ascites, poor liver function, '! MELD)

- Difficult to treat SBP:

a) Community acquired with risk of developing renal dysfunction: cefotaxime and albumin

b) Nosocomial: high risk of ESBL/MRSA/ multi resistant bacteria, associated with increased mortality (1st line of treatment should be ? carbapenem ? glycopeptides)

- Local flora and its sensitivity pattern are important in choosing the appropriate antibiotics in nosocomial SBP

- All patients with one episode of SBP should be considered for liver transplantation

- Alternative strategies for long term prophylaxis without development of bacterial resistance are needed

\section{References}

1. Caly WR, Strauss E. A prospective study of bacterial infections in patients with cirrhosis. J Hepatol. 1993;18:353-8

2. Evans LT, Kim WR, Poterucha JJ, Kamath PS. Spontaneous bacterial peritonitis in asymptomatic outpatients with cirrhotic ascites. Hepatology. 2003;37:897-901.

3. Nobre SR, Cabral JE, Gomes JJ, Leitão MC. In-hospital mortality in spontaneous bacterial peritonitis: a new predictive model. Eur J Gastroenterol Hepatol. 2008;20:1176-81.

4. Wiest R, Krag A, Gerbes A. Spontaneous bacterial peritonitis: recent guidelines and beyond. Gut. 2012;61:297-310.

5. Rimola A, García-Tsao G, Navasa M, Piddock LJ, Planas R, Bernard B, Inadomi JM. Diagnosis, treatment and prophylaxis of spontaneous bacterial peritonitis: a consensus document. International Ascites Club. J Hepatol. 2000;32:142-53.

6. Runyon BA; AASLD Practice Guidelines Committee. Management of adult patients with ascites due to cirrhosis: an update. Hepatology. 2009;49:2087-107.

7. European Association for the Study of the Liver. EASL clinical 
practice guidelines on the management of ascites, spontaneous bacterial peritonitis, and hepatorenal syndrome in cirrhosis. $J$ Hepatol. 2010;53:397-417.

8. Wiest R, Garcia-Tsao G. Bacterial translocation (BT) in cirrhosis. Hepatology. 2005;41:422-33.

9. Nousbaum JB, Cadranel JF, Nahon P, Khac EN, Moreau R et al. Diagnostic accuracy of the Multistix 8 SG reagent strip in diagnosis of spontaneous bacterial peritonitis. Hepatology. 2007; 45:1275-81.

10. Bruns T, Sachse S, Straube E, Assefa S, Herrmann A et al. Identification of bacterial DNA in neutrocytic and non neutrocytic cirrhotic ascites by means of a multiplex polymerase chain reaction. Liver Int. 2009;29:1206-14.

11. Parsi MA, Saadeh SN, Zein NN, Davis GL, Lopez R et al. Ascitic fluid lactoferrin for diagnosis of spontaneous bacterial peritonitis. Gastroenterology. 2008;135:803-7.

12. Ortiz J, Soriano G, Coll P, Novella MT, Pericas R et al. Early microbiologic diagnosis of spontaneous bacterial peritonitis with BacT/ALERT. J Hepatol. 1997;26:839-44.

13. Castellote J, Xiol X, Verdaguer R, Ribes J, Guardiola J et al. Comparison of two ascitic fluid culture methods in cirrhotic patients with spontaneous bacterial peritonitis. Am J Gastroenterol. 1990;85:1605-8.

14. Andreu M, Sola R, Sitges-Serra A, Alia C, Gallen M et al. Risk factors for spontaneous bacterial peritonitis in cirrhotic patients with ascites. Gastroenterology. 1993;104:1133-8.

15. Such J, Guarner C, Enriquez J, Rodriguez JL, Seres I et al. Low C3 in cirrhotic ascites predisposes to spontaneous bacterial peritonitis. J Hepatol. 1988;6:80-4.

16. Obstein KL, Campbell MS, Reddy KR, Yang YX. Association between model for end-stage liver disease and spontaneous bacterial peritonitis. Am J Gastroenterol. 2007;102:2732-6.

17. Guarner C, Solà R, Soriano G, Andreu M, Novella MT et al. J. Risk of a first community-acquired spontaneous bacterial peritonitis in cirrhotics with low ascitic fluid protein levels.Gastroenterology. 1999;117:414-9.

18. Bernard B, Cadranel JF, Valla D, Escolano S, Jarlier V et al. Prognostic significance of bacterial infection in bleeding cirrhotic patients: a prospective study. Gastroenterology. 1995;108:1828-34.

19. Gäbele E, Mühlbauer M, Paulo H, Johann M, Meltzer C et al. Analysis of monocyte chemotactic protein-1 gene polymorphism in patients with spontaneous bacterial peritonitis. World $J$ Gastroenterol. 2009;15:5558-62.

20. Bruns T, Peter J, Reuken PA, Grabe DH, Schuldes SR et al. NOD2 gene variants are a risk factor for culture-positive spontaneous bacterial peritonitis and monomicrobial bacterascites in cirrhosis. Liver Int. 2012;32:223-30.

21. Nischalke HD, Berger C, Aldenhoff K, Thyssen L, Gentemann
$\mathrm{M}$ et al. Toll-like receptor (TLR) 2 promoter and intron 2 polymorphisms are associated with increased risk for spontaneous bacterial peritonitis in liver cirrhosis. J Hepatol. 2011;55:1010-6.

22. Lo WK, Chan WW. Proton Pump Inhibitor Use and the Risk of Small Intestinal Bacterial Overgrowth: A Meta-analysis. Clin Gastroenterol Hepatol. 2012 Dec 24. doi:pii: S15423565(12)01511-X. 10.1016/j.cgh.2012.12.011. [Epub ahead of print]

23. Senzolo M, Cholongitas E, Burra P, Leandro G, Thalheimer U et al. beta-Blockers protect against spontaneous bacterial peritonitis in cirrhotic patients: a meta-analysis. Liver Int. 2009;29:1189-93.

24. Umgelter A, Reindl W, Miedaner M, Schmid RM, Huber W. Failure of current antibiotic first-line regimens and mortality in hospitalized patients with spontaneous bacterial peritonitis. Infection. 2009;37:2-8.

25. Ariza X, Castellote J, Lora-Tamayo J, Girbau A, Salord S et al. Risk factors for resistance to ceftriaxone and its impact on mortality in community, healthcare and nosocomial spontaneous bacterial peritonitis. J Hepatol. 2012;56:825-32.

26. Piroth L, Pechinot A, Minello A, Jaulhac B, Patry I et al. Bacterial epidemiology and antimicrobial resistance in ascitic fluid: a 2year retrospective study. Scand J Infect Dis. 2009;41:847-51.

27. Arvaniti V, D'Amico G, Fede G, Manousou P, Tsochatzis E et al. Infections in patients with cirrhosis increase mortality four-fold and should be used in determining prognosis. Gastroenterology. 2010;139:1246-56

28. Runyon BA, McHutchison JG, Antillon MR, Akriviadis EA, Montano AA. Short-course versus long-course antibiotic treatment of spontaneous bacterial peritonitis. A randomized controlled study of 100 patients. Gastroenterology. 1991;100:1737-42.

29. Sort P, Navasa M, Arroyo V, Aldeguer X, Planas R et al. Effect of intravenous albumin on renal impairment and mortality in patients with cirrhosis and spontaneous bacterial peritonitis. $N$ Engl $J$ Med. 1999;341:403-9.

30. Chen TA, Tsao YC, Chen A, Lo GH, Lin CK et al. Effect of intravenous albumin on endotoxin removal, cytokines, and nitric oxide production in patients with cirrhosis and spontaneous bacterial peritonitis. Scand J Gastroenterol. 2009;44:619-25.

31. Ginés P, Rimola A, Planas R, Vargas V, Marco F et al. Norfloxacin prevents spontaneous bacterial peritonitis recurrence in cirrhosis: results of a double-blind, placebo-controlled trial. Hepatology. 1990;12:716-24.

32. Fernández J, Navasa M, Planas R, Montoliu S, Monfort D et al. Primary prophylaxis of spontaneous bacterial peritonitis delays hepatorenal syndrome and improves survival in cirrhosis. Gastroenterology. 2007;133:818-24. 